\title{
Hospitals as health factories and the coronavirus epidemic
}

\author{
Giorgina Barbara Piccoli ${ }^{1,2}$
}

Published online: 21 March 2020

(c) Italian Society of Nephrology 2020

Keywords SARS-CoV-2 infection · Dialysis $\cdot$ Hospital wards $\cdot$ Hospital architecture

In this issue, on behalf of the Italian Society of Nephrology, a group of nephrologists, mainly from Lombardy, the Italian region most affected by the epidemics, proposes suggestions on the management of SARS-CoV-2 infection in the dialysis ward, one of the most vulnerable hospital wards [1].

This effort, based upon direct experience, scientific data (still few), and knowledge from previous epidemics, is highly welcome, in a moment in which uncertainties are more common than certitudes, and Europe struggles to be prepared to face the unknown.

Amidst anxiety, hysteria and serious concerns, the Coronavirus epidemics should indeed lead to number of reflections on the organisation of healthcare and the way contemporary medicine has lost sight of some diseases, such as infectious ones, that were, probably prematurely, seen as diseases of the past.

The spread of this new, poorly understood infection is indeed a challenge: as far as we know, contamination leads to an overt infection in about half of cases, with a wide range of signs and symptoms, from a mild flu-like illness, to a severe, potentially lethal pneumonia. As far as we presently know, re-infection is possible and contamination can occur in the pre-symptomatic stage or the disease can be transmitted by non-symptomatic carriers [2].

Since PCR virus testing capabilities are widely available, concerns regarding the spread of panic, are an important part of managing the outbreak. In settings where the test is restricted to severely symptomatic cases, knowledge about preclinical diffusion is limited and it may not be possible to efficiently organise preventive measures. Different scientific

Giorgina Barbara Piccoli

gbpiccoli@yahoo.it

1 Nephrologie, Centre Hospitalier Le Mans, Avenue Roubillard 196, 72000 Le Mans, France

2 Dipartimento Di Scienze Cliniche E Biologiche, Università Di Torino, Turin, Italy bodies have given conflicting advice, and the lack of availability of efficacious protective equipment is complicating the picture [3].

The Pandora's box has been opened [4]. In an attempt to limit the spread of the disease, schools and factories have been closed, congresses and meetings are being cancelled and people are being advised to avoid contact with others, including shaking hands.

More specifically in medicine, a common indication is to limit hospital access to patients with severe clinical problems, or those who, like dialysis patients, require regular hospital treatment.

Interestingly, this indication has come at a moment when many hospitals, including ours, are reorganising and "industrializing" out-patient care, building large open spaces, where multiple specialists will furnish hundreds, or perhaps even thousands of consultations per day.

Having access to shared services, provided by secretaries, nurses, aides and housekeeping personnel is obviously appealing from an economic point of view. The advantages from the clinical point of view are less clear: a factory-like hospital is intuitively far from the ideal of personalised medicine, and such a system may prove incapable of providing specific, personalised contact with the caregivers, a point that is often important for chronic patients.

But the history of SARS-CoV-2 has shown us something else: in this attempt to "rationalize" our activities, we have forgotten about infectious diseases. Having nineteen posts directing patients towards 120 consultation rooms mean that every day, if the system works at full efficiency, up to over one thousand patients wait in line, sit on a bench, enter a room, go back to the secretary, make another appointment, and finally wait for transportation to go home.

We are now slowly realizing that this "super-efficient", factory-like, program is incompatible with the periodic occurrence of epidemics, a scenario many experts consider likely, whose consequences will probably be more severe 


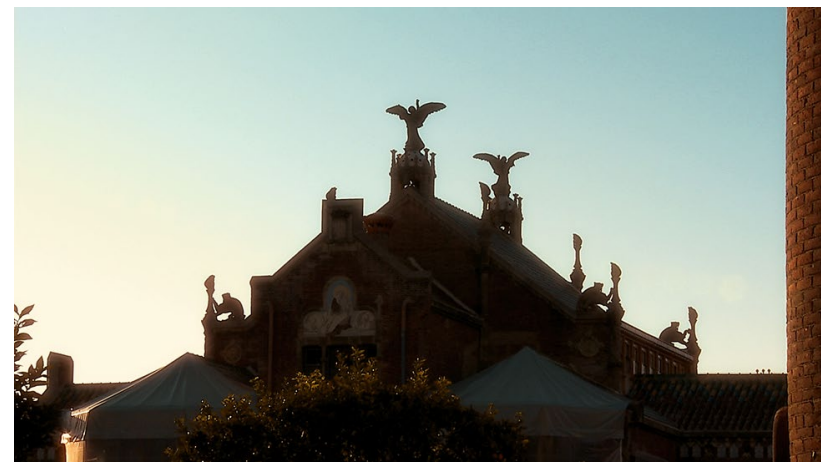

Fig. 1 The beauty (and not only the devil) in the details

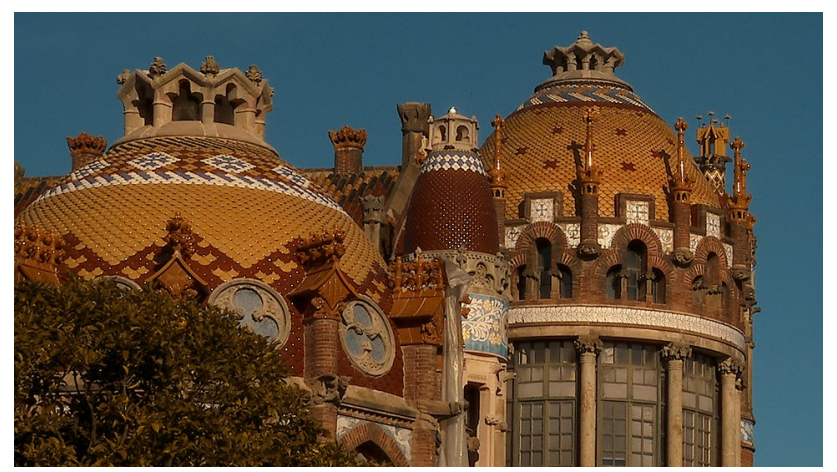

Fig. 2 Architectural details of the pavilions of the Hospital de la Santa Creu i Sant Pau. Courtesy of Gilberto Richiero

because of globalization [5]. While economies of scale seem to favour "hospital factories", events like the Covid19 infection risk compromising their ability to function, and could ultimately cause large economic losses, by making it impossible to differentially modulate the provision of care when limiting human-to-human contact is necessary.

We have definitely not won the fight against infectious diseases, but we have probably forgotten about them too soon. In a high-technology setting, it is all too easy to forget the overwhelming, often dark power of nature; Fukushima, is a clear example.

It is therefore with a bit of nostalgia, a great deal of admiration and some reflections on how hospitals are now being built that we look back on the "pavilion hospitals" of the early twentieth century., some of exceptional beauty, first of all the UNESCO World Heritage Site, Hospital de la Santa Creu i Sant Pau in Barcelona (Figs. 1, 2). This hospital, designed by the Catalan architect Lluís Domènech i Montaner, embodies an approach to healthcare that we would do well to reconsider today. The beauty of its decorations was thought to help the healing process and the trees in its large garden were chosen for their association with phytotherapeutic properties.
Most importantly, at a time when infectious diseases were the major cause of death, each disease was treated in a separate pavilion, with separate entrances and exits which made it possible to prevent contact between patients affected by different illnesses.

There were a number of similar hospitals in Europe. The Royal Navy Hospital, Stonehouse in Plymouth, England; the Lariboisière Hospital in Paris and the Édouard Herriot Hospital in Lyon, France; or the Amedeo di Savoia Hospital in Turin, Italy are just some examples of this architectural period, whose style was progressively abandoned in favour of the "block hospital" [6].

Should we return to the idea that beauty-in the natural settings surrounding hospitals and the buildings themselves-can contribute to healing?

Probably not, even though this may be appealing.

However, our experience with the new Coronavirus could serve as a healthy paradigm shock to our healthcare system, leading us to reconsider the profound risks generated by the frenetic search for hospital productivity and remind us that our job is caring, as human beings, for other human beings, that benefit from being treated by people they know (secretaries and nurses as well as doctors), in a familiar setting.

Facilitating the management of infectious contacts is probably just one of the advantages.

Acknowledgements To the Centre Hospitalier Le Mans for supporting language editing. To Susan Finnel for her careful language review. To Gil Richiero for the images of the Hospital de la Santa Creu i Sant Pau.

Funding None.

\section{Compliance with ethical standards}

Conflict of interest None.

Ethical standard Not required for this paper.

\section{References}

1. Rombolà et al (to be completed according to the final manuscript)

2. Guan WJ, Ni ZY, Hu Y et al; China Medical Treatment Expert Group for Covid-19 (2020) Clinical characteristics of coronavirus disease 2019 in China. N Engl J Med. https://doi.org/10.1056/ NEJMoa2002032(epub ahead of print)

3. Chang, Xu H, Rebaza A, Sharma L, Dela Cruz CS (2020) Protecting health-care workers from subclinical coronavirus infection. Lancet Respir Med 8(3):e13. https://doi.org/10.1016/S2213 -2600(20)30066-7

4. Morens DM, Daszak P, Taubenberger JK (2020) Escaping Pandora's box-another novel coronavirus. N Engl J Med. https://doi. org/10.1056/NEJMp2002106(epub ahead of print)

5. Peeri NC, Shrestha N, Rahman MS, Zaki R, Tan Z, Bibi S, Baghbanzadeh M, Aghamohammadi N, Zhang W, Haque U (2020) The SARS, MERS and novel coronavirus (COVID-19) epidemics, 
the newest and biggest global health threats: what lessons have we learned? Int J Epidemiol. https://doi.org/10.1093/ije/dyaa0 33(epub ahead of print)

6. Bates V (2018) 'Humanizing' healthcare environments: architecture, art and design in modern hospitals. Des Health (Abingdon) 2(1):5-19
Publisher's Note Springer Nature remains neutral with regard to jurisdictional claims in published maps and institutional affiliations. 